\title{
Long Memory Analysis in DNA Sequences
}

\author{
S. R. C. Lopes and M. A. Nunes \\ Instituto de Matemática, UFRGS, Porto Alegre, Brazil
}

\begin{abstract}
Our goal in this work is to construct empirical confidence intervals for the fractional parameter $d$ in $\operatorname{ARFIMA}(0, d, 0)$ processes. Through these confidence intervals one can compare several estimators for $d$ to decide which one is the best estimation method related to long memory time series. We use a FORTRAN routine that simulates random time series to latter perform an analysis for detecting long memory. We also apply the methodology to real DNA sequences to evaluate the efficiency of our method in the construction of these confidence intervals.
\end{abstract}

Key words: Long Memory, DNA Sequences, Empirical Confidence Intervals.

\section{Introduction}

A time series is a register of values for a certain random variable, measured in different discrete times. For instance, the daily temperature of one city measured at the same hour, during some interval of time, where the previous measure is related to the latter one.

We shall use $\left\{X_{t}\right\}_{t \in T}$ to denote a stochastic process in time $t \in T$, where $T$ is an index set. For each $t \in T, X_{t}$ is a random variable.

Recently, many researchers in time series analysis are studying the ones with long memory characteristics, that is, time series with significant dependence between observations apart for a long period of time. The goal here is to use these characteristics to construct an adequate model for the time series.

According to the works [1], [2], and [3], DNA sequences show long memory, and the goal here is to properly estimate the parameter that describes this characteristic. In order to do this, we consider the ARFIMA (autoregressive fractionally integrated moving average) models with $(p, d, q)$ parameters where the fractional parameter $d$ measures the long memory property when $d \in$ $(0.0,0.5)$, and $p$ and $q$ are the orders of the autoregressive and moving average 
processes, respectively. We shall consider five different estimation methods for $d$.

This paper is organized as follows: Section 2 describes the stochastic processes with long memory characteristic treating, particularly, the case of ARFIMA $(p, d, q)$ models. In Section 3 we present the chemical structure of a DNA sequence. An explanation of the different estimators for $d$ is given in Section 4. In Section 5 we construct the empirical confidence intervals based on each estimator proposed in the previous section. We analyze a real DNA sequence in Section 6, estimating the value of $d$ through the proposed estimator methods obtaining their confidence intervals. Section 7 concludes this paper.

\section{Long Memory Models}

In this section we present the $\operatorname{ARFIMA}(p, d, q)$ model (also called Fractional ARIMA model) and some related theoretical results. Models that includes fractional differentiation $d$ in the interval $(0.0 ; 0.5)$ are able to represent any time series that shows persistence, also known by long memory property (see [4] for a complete study of these models). Initial studies of time series with long memory characteristics were given by [5]. ARFIMA processes first appeared in [6] and [7] and are a generalization of the ARMA and ARIMA models. The author of [8] was the pioneer in the application of long memory in hydrological time series.

Persistence or long memory property has been observed in time series from different fields such as meteorology, astronomy, hydrology, and economy. One can characterize the persistence by two different forms:

- in time domain, the autocorrelation function $\rho_{X}(\cdot)$ decays hyperbolically to zero, that is, $\rho_{X}(k) \simeq k^{2 d-1}$, when $k \rightarrow \infty$.

- in frequency domain, the spectral density function $f_{X}(\cdot)$ is unbounded when the frequency is near zero, that is, $f_{X}(w) \simeq w^{-2 d}$, when $w \rightarrow 0$.

One of the models that can describe the persistence is the so called ARFIMA $(p, d, q)$ processes.

$\operatorname{ARFIMA}(p, d, q)$ Process

Definition 1 A stochastic process $\left\{X_{t}\right\}_{t \in \mathbb{Z}}$ is Gaussian if, for any set of $t_{1}$, $t_{2}, \cdots, t_{n} \in \mathbb{Z}$, the random variables $X_{t_{1}}, X_{t_{2}}, \cdots, X_{t_{n}}$ have a $n$-dimensional normal distribution. 
We observe that weakly stationary process $\left\{X_{t}\right\}_{t \in \mathbb{Z}}$ does not need to be strongly stationary. However, any weakly stationary Gaussian process will be also strongly stationary (see [9]).

Definition 2 The process $\left\{\varepsilon_{t}\right\}_{t \in \mathbb{Z}}$ is said to be a white noise process with zero mean and variance $\sigma_{\varepsilon}^{2}$, denoted by $\varepsilon_{t} \sim W N\left(0, \sigma_{\varepsilon}^{2}\right)$, if

$$
\mathbb{E}\left(\varepsilon_{t}\right)=0, \quad \operatorname{Var}\left(\varepsilon_{t}\right)=\mathbb{E}\left(\varepsilon_{t}^{2}\right)=\sigma_{\varepsilon}^{2}, \quad \text { and } \quad \gamma_{\varepsilon}(k)=\left\{\begin{array}{l}
\sigma_{\varepsilon}^{2}, k=0 \\
0, \quad k \neq 0
\end{array}\right.
$$

Definition 3 Let $\left\{\varepsilon_{t}\right\}_{t \in \mathbb{Z}}$ be a white noise process with zero mean and variance $\sigma_{\varepsilon}^{2}>0$, and $\mathcal{B}$ the backward-shift operator, i.e., $\mathcal{B}^{k}\left(X_{t}\right)=X_{t-k}$. If $\left\{X_{t}\right\}_{t \in \mathbb{Z}}$ is a linear process satisfying

$$
\phi(\mathcal{B})(1-\mathcal{B})^{d} X_{t}=\theta(\mathcal{B}) \varepsilon_{t}, \quad t \in \mathbb{Z},
$$

where $d \in(-0.5 ; 0.5), \phi(\cdot)$, and $\theta(\cdot)$ are polynomials of degree $p$, and $q$ respectively, given by

$$
\begin{aligned}
\phi(\mathcal{B}) & =1-\phi_{1} \mathcal{B}-\cdots-\phi_{p} \mathcal{B}^{p} \\
\theta(\mathcal{B}) & =1-\theta_{1} \mathcal{B}-\cdots-\theta_{q} \mathcal{B}^{q}
\end{aligned}
$$

where $\phi_{i}, 1 \leqslant i \leqslant p$, and $\theta_{j}, 1 \leqslant j \leqslant q$, are real constants, then $\left\{X_{t}\right\}_{t \in \mathbb{Z}}$ is called general fractional differentiation $\operatorname{ARFIMA}(p, d, q)$ process, where $d$ is the degree or fractional differentiation parameter.

The term $(1-\mathcal{B})^{d}$, for $d \in \mathbb{R}$, is defined through the binomial expansion

$$
(1-\mathcal{B})^{d}=\sum_{k=0}^{\infty}\left(\begin{array}{l}
d \\
k
\end{array}\right)(-\mathcal{B})^{k}=1-d \mathcal{B}-\frac{d}{2 !}(1-d) \mathcal{B}^{2} \cdots
$$

If $d \in(-0.5 ; 0.5)$, then $\left\{X_{t}\right\}_{t \in \mathbb{Z}}$ is a stationary, and an invertible process (see Theorem 4 below, for the case where $p=0=q)$.

The most important characteristic of an $\operatorname{ARFIMA}(p, d, q)$ process is the property of long dependence, when $d \in(0.0 ; 0.5)$, short dependence, when $d=0$, and intermediate dependence, when $d \in(-0.5 ; 0.0)$. In this work we analyze only processes with long memory property. 
In this work we consider ARFIMA processes where $p$ and $q$ are both equal to zero. The $\operatorname{ARFIMA}(0, d, 0)$ processes are given by

$$
(1-\mathcal{B})^{d} X_{t}=\varepsilon_{t}, \text { for all } t \in \mathbb{Z}
$$

Important properties for $\operatorname{ARFIMA}(0, d, 0)$ processes can be found in [7]. The following theorem supplies the main properties for these processes.

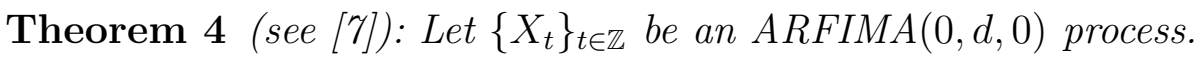

(a) When $d<0.5,\left\{X_{t}\right\}_{t \in \mathbb{Z}}$ is a stationary process with an infinite moving average representation given by

$$
X_{t}=\psi(\mathcal{B}) \varepsilon_{t}=\sum_{k=0}^{\infty} \psi_{k} \varepsilon_{t-k}
$$

where

$$
\psi_{k}=\frac{d(1+d) \cdots(k-1+d)}{k !}=\frac{(k+d-1) !}{k !(d-1) !} .
$$

When $k \rightarrow \infty, \quad \psi_{k} \simeq \frac{k^{d-1}}{(d-1) !}$.

(b) When $d>-0.5,\left\{X_{t}\right\}_{t \in \mathbb{Z}}$ is an invertible process with an infinite autoregressive representation given by

$$
\pi(\mathcal{B}) X_{t}=\sum_{k=0}^{\infty} \pi_{k} X_{t-k}=\varepsilon_{t}
$$

where

$$
\pi_{k}=\frac{-d(1-d) \cdots(k-1-d)}{k !}=\frac{(k-d-1) !}{k !(-d-1) !} .
$$

When $k \rightarrow \infty, \pi_{k} \simeq \frac{k^{-d-1}}{(-d-1) !}$.

In items (c), (d) and (e) below, we assume that $d \in(-0.5 ; 0.5)$.

(c) The spectral density function of $\left\{X_{t}\right\}_{t \in \mathbb{Z}}$ is given by

$$
f_{X}(w)=\left[2 \sin \left(\frac{w}{2}\right)\right]^{-2 d}, \text { for } 0<w \leq \pi .
$$

When $w \simeq 0, f_{X}(w) \simeq w^{-2 d}$. 
(d) The autocovariance function of $\left\{X_{t}\right\}_{t \in \mathbb{Z}}$ is given by

$$
\gamma_{X}(k)=\frac{(-1)^{k}(-2 d) !}{(k-d) !(-k-d) !}
$$

and the autocorrelation function is given by

$$
\rho_{X}(k)=\frac{(-d) !(k+d-1) !}{(d-1) !(k-d) !}, \text { for all } k \in \mathbb{Z}
$$

When $k \rightarrow \infty, \rho_{X}(k) \simeq \frac{(-d) !}{(d-1) !} k^{2 d-1}$.

(e) The partial autocorrelation function of $\left\{X_{t}\right\}_{t \in \mathbb{Z}}$ is given by

$$
\phi_{X}(k, k)=\frac{d}{k-d}, \text { for all } k \in \mathbb{N}
$$

Remark 5 For $d>0$ the autocorrelation function $\rho_{X}(k)$ has hyperbolic decay when $k$ increases, and the spectral density function is unbounded for frequencies near to zero frequency demonstrating the capability of the model to show persistence.

Remark 6 If $\left\{X_{t}\right\}_{t \in \mathbb{Z}}$ is defined by the expression (2), then its spectral density function is given by

$$
f_{X}(w)=f_{U}(w)\left[2 \sin \left(\frac{w}{2}\right)\right]^{-2 d}, \text { for all } 0<w \leq \pi
$$

where $f_{U}(\cdot)$ denotes the spectral density function of an $A R M A(p, q)$ process, $U_{t}$, given by

$$
(1-\mathcal{B})^{d} X_{t}=U_{t}, \text { for all } t \in \mathbb{Z}
$$

\section{DNA Chemical Structure}

The DNA is the deoxyribonucleic acid. The DNA ribbons are long polymers made of millions of nucleotides connected some to the others. Individually, nucleotides are quite simple, consisting of three distinct parts: one of the four nitrogenized bases, a deoxyribose (a sugar of 5 carbons), and a phosphate group. 

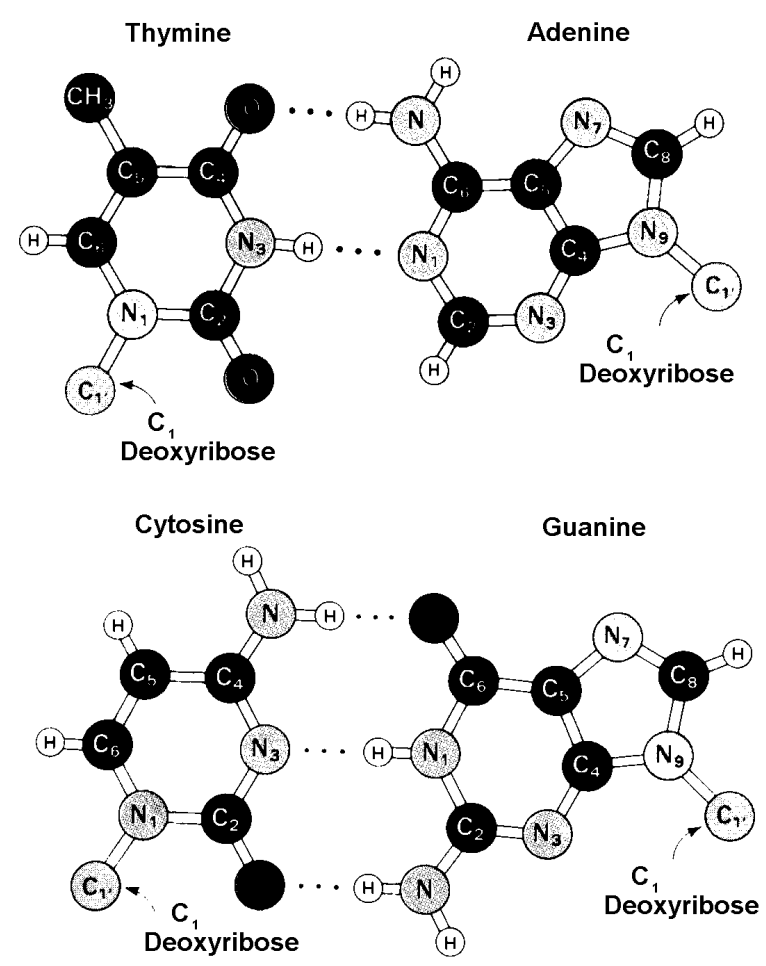

Fig. 1. Illustration showing how the bases pairs are connected by hydrogen bridges.

The denomination of the nucleotides depends on the nitrogenized basis that composes them. A DNA sequence is composed by four nucleotides called as adenine, guanine, cytosine, and thymine, denoted by the capital letters A, G, $\mathrm{C}$, and T, respectively. (Note: In this work, the words nucleotide and basis will be used to represent the same thing, i.e., a nucleotide).

Adenine and guanine, a two ring composed molecules, are classified as purines. Cytosine and thymine are classified as pyrimidines and they are molecules formed by only one ring. One purine connects to one pyrimidine in a DNA sequence to form a pair of bases. Adenine, and thymine are connected to each other to form a pair of A-T bases, while guanine, and cytosine form a pair of $\mathrm{G}-\mathrm{C}$ bases. The bases remain joined for weakly hydrogen bridges, and these hydrogen bridges are responsible in order to maintain the structure of a double helix of the DNA sequence (see Figure 1).

\section{Autocorrelation Function in DNA Sequences}

It is not evident what makes the DNA sequence to present long memory characteristics, but they do so, and this may be related to the evolution's mechanism since the growth of the first form of life on Earth.

When life appeared in our planet, billions of years ago, it appeared from the 
random combinations in the seas in formation. Passing the time by, for natural processes, these particles had been increasing, and combining, to generate more complex, and adaptable organisms to the environment; this increasing or "elongation" occurred through the so called oligonucleotide duplication or duplication of the genes process in which a segment was removed, and some times duplicate, after being reinserted in the original sequence. It is clear that such process was not perfect, and from these small mutations the evolution was made.

Introns are nucleotide sequences that do not "generate" proteins; in contrast with exons, that do generate them. Sometimes called by "junk genes", introns seem not to have any function in the genetic sequence. However, nowadays the biologists have doubts of this (see [10]), and they do believe that introns possess important functions in the mechanism of the evolution. For unknown reasons, introns do not suffer many changes as exons during the duplication, provoking long memory property more evident through them. Our goal here is to study the parameter of long memory in time series consisting by introns and exons.

\section{Random Walk}

One can consider several different ways to construct a random walk from DNA sequences (see, for instance, [1], [11], and [12]). Here, in this work, the classification in purines, and pyrimidines was chosen because its better detection of the long dependence property in DNA sequences (see, for instance, [1] and [11]).

In order to study the properties of a DNA sequence we construct a random walk in one dimension, based on this classification. In a DNA sequence, if in the position $i$ one finds a pyrimidine, we give one step upward, otherwise, if a purine is found we give one step downward (see [11]). Therefore, we define the function $g(\cdot)$ such that

$$
g(i)= \begin{cases}+1, & \text { if } i=\text { pyrimidine } \\ -1, & \text { if } i=\text { purine }\end{cases}
$$

After $t$ positions, the random walk is the addition of the $g(i)$ steps up to position $t$, that is,

$$
X_{t}=\sum_{i=1}^{t} g(i)
$$

A FORTRAN routine was written to identify the bases, from a standard text 
archive, determining the steps, and the random walk. We have then a time series $\left\{X_{t}\right\}_{t=1}^{n}$, and we proceed with a long memory analysis based on this data. In general, the time series $\left\{X_{t}\right\}_{t=1}^{n}$ is a sample from a non-stationary stochastic process. In order to obtain a stationary time series we take a first difference of it denoted by

$$
Y_{t}=X_{t}-X_{t-1}=(1-\mathcal{B}) X_{t}, \text { for } t \in \mathbb{N},
$$

where $\mathcal{B}$ is the backward-shift operator. Our goal is to study the long memory property of the stochastic process $\left\{Y_{t}\right\}_{t \in \mathbb{N}}$ based on $\operatorname{ARFIMA}(0, d, 0)$ processes. We want to estimate properly the parameter $d$ when $d \in(0.0 ; 0.5)$. We recall that if $\hat{d}_{X}=1.2$ is an estimator of $d$, under the stochastic process $\left\{X_{t}\right\}_{t \in \mathbb{N}}$, then $\hat{d}_{Y} \approx 0.2$, under the stochastic process $\left\{Y_{t}\right\}_{t \in \mathbb{N}}$ (see [13]). For the estimation of parameter $d$ when $d \in(0.5,1.5)$ we refer the reader to [14].

\section{Fractional Parameter Estimation}

We now summarize some methods for the estimation of $d$ : the regression methods using the periodogram function $\left(\hat{d}_{G P H}\right)$, proposed by Geweke, and Porter-Hudak in [15], and the smoothed version of the periodogram function $\left(\hat{d}_{S P R}\right)$, proposed by Reisen in [16]; the estimator proposed by Robinson in [17] $\left(\hat{d}_{R P}\right)$ based on the Geweke, and Porter-Hudak's method, where the number of regressors in the regression equation starts from $l>1$ instead of one and its smoothed version proposed by this work $\left(\hat{d}_{R S P}\right)$; and the approximated maximum likelihood estimator $\left(\hat{d}_{W}\right)$, proposed by Fox, and Taqqu in [18], based on an idea of [19].

Estimator $\hat{d}_{G P H}$

Consider the set of Fourier frequencies $w_{j}=\frac{2 \pi j}{n}, j=1, \cdots,[n / 2]$, where $n$ is the sample size and $[x]$ means the integer part of $x$. By taking the logarithm of the spectral density function $f_{X}(\cdot)$, and adding $\ln f_{U}(0)$, and $\ln I\left(w_{j}\right)$ to both sides of the expression (4) we have

$$
\ln I\left(w_{j}\right)=\ln f_{U}(0)-d \ln \left[2 \sin \left(\frac{w_{j}}{2}\right)\right]^{2}+\ln \left[\frac{f_{U}\left(w_{j}\right)}{f_{U}(0)}\right]+\ln \left[\frac{I\left(w_{j}\right)}{f_{X}\left(w_{j}\right)}\right]
$$

where $I(\cdot)$ is the periodogram function. 
The estimator of $d$ is given by

$$
\hat{d}_{G P H}=-\frac{\sum_{j=1}^{g(n)}\left(x_{j}-\bar{x}\right)\left(y_{j}-\bar{y}\right)}{\sum_{j=1}^{g(n)}\left(x_{j}-\bar{x}\right)^{2}},
$$

where $g(n)=n^{\alpha}, 0<\alpha<1$ (see [15]), $y_{j}=\ln I\left(w_{j}\right), x_{j}=\ln \left[2 \sin \left(w_{j} / 2\right)\right]^{2}$, and $\bar{x}=\frac{1}{g(n)} \sum_{j=1}^{g(n)} x_{j}$.

Estimator $\hat{d}_{S P R}$

The regression estimator $\hat{d}_{S P R}$ is obtained by replacing the periodogram function in the expression $(7)$ by the smoothed periodogram function, $f_{s}(\cdot)$, with the Parzen lag window. The parameter $m$ in the lag window generator, usually referred to as the truncation point, is a function of the sample size chosen as $m=n^{\beta}$, for $0<\beta<1$. The paper [16] shows that $\hat{d}_{S P R}$ is given by the same expression as in (8), where now $y_{j}=\ln f_{s}\left(w_{j}\right)$, for $j=1, \cdots, g(n)$. The value of $g(n)$ is chosen as in the $\hat{d}_{G P H}$ method.

Estimators $\hat{d}_{R P}$ and $\hat{d}_{R S P}$

We also consider the estimator proposed in [17], and its smoothed variation proposed by this work. The first one, denoted by $\hat{d}_{R P}$, is a modified version of the estimator $\hat{d}_{G P H}$, where the number of regressors $g(n)$, in the expression $(7)$, starts from $l>1$ instead of one (see [17]). The second one, denoted by $\hat{d}_{R S P}$, uses the smoothed version of the periodogram function instead of the periodogram itself.

Estimator $\hat{d}_{W}$

This estimator involves the function

$$
Q(\eta)=\int_{-\pi}^{\pi} \frac{I(w)}{f_{X}(w ; \eta)} d w
$$

where $f_{X}(\cdot ; \eta)$ is the spectral density function of the $\left\{X_{t}\right\}_{t \in \mathbb{N}}$, and $\eta$ denotes the vector of unknown parameters. The $\hat{d}_{W}$ estimator is the value of $\eta$ which minimizes the function $Q(\cdot)$ (see [19]). When we are dealing with the situation where $p=0=q, \eta$ is given only by the parameter $d$. For computational purposes, it is easier to minimize the function 


$$
\mathcal{L}_{n}(\eta)=\frac{1}{2 n} \sum_{j=1}^{n-1}\left\{\ln f_{X}\left(w_{j} ; \eta\right)+\frac{I\left(w_{j}\right)}{f_{X}\left(w_{j} ; \eta\right)}\right\}
$$

instead of $Q(\cdot)$, where $w_{j}$ are the Fourier frequencies, for $j=1, \cdots, n-1$. For general $\operatorname{ARFIMA}(0, d, 0)$ Gaussian processes Fox, and Taqqu have shown in [18] that the maximum likelihood estimator of $d$ is strongly consistent, and asymptotically normally distributed.

\section{Confidence Intervals Construction}

In this section we describe the method used for the construction of the empirical confidence intervals based on the estimators proposed on the previous section. We follow the ideas in the works [20] and [21].

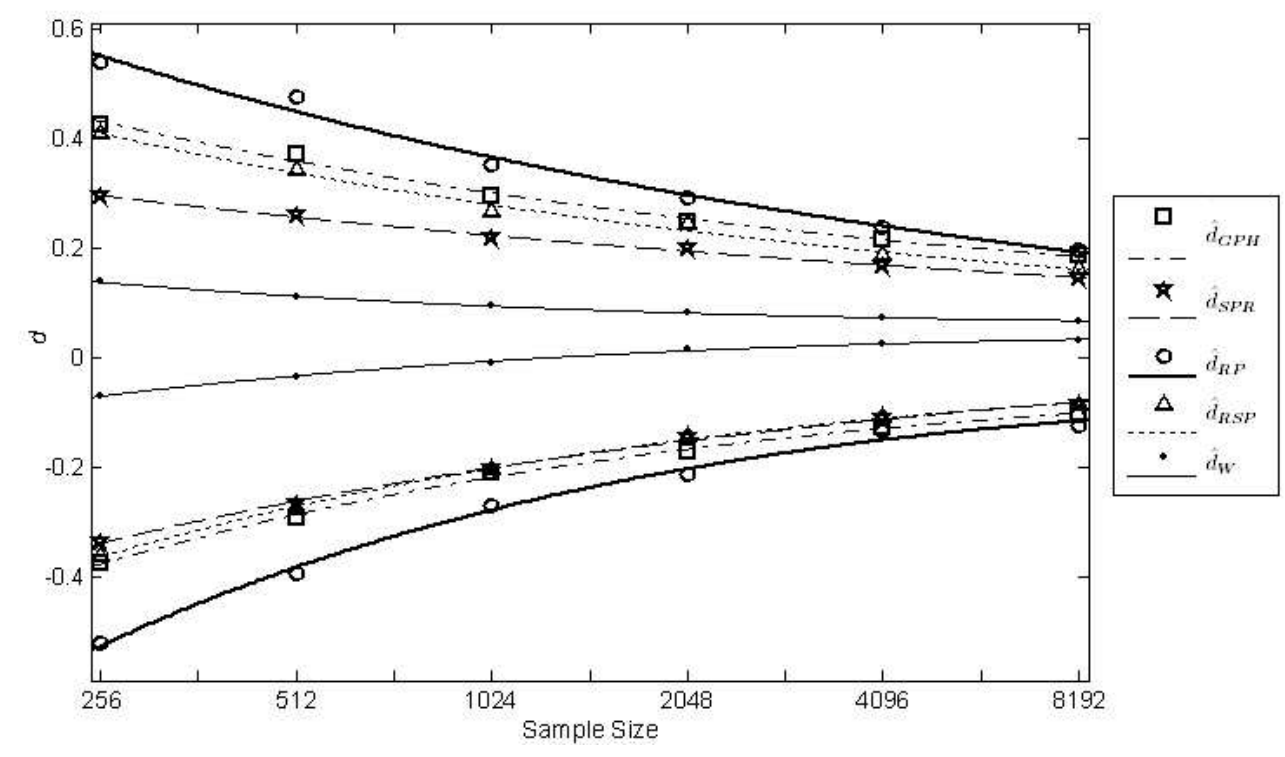

Fig. 2. Confidence interval for $d=0.05$ at $95 \%$ based on the five considered estimation methods and on six different sample sizes.

We do not use the asymptotic theory of all estimators, instead we wrote a FORTRAN routine using the method proposed in [8] to generate an $\operatorname{ARFIMA}(0, d, 0)$ process. The steps of this algorithm are given as follows:

(1) Calculate the partial autocorrelation function $\varphi_{X}(j, j)$.

(2) Generate a random variable $\mathcal{N}(0,1)$, through the subroutine RNNOR ${ }^{1}$, of size $n$, to simulate a Gaussian white noise $\left\{\varepsilon_{t}\right\}_{t \in \mathbb{Z}}$ process.

$\overline{1}$ This subroutine belongs to the IMSL FORTRAN library and generates pseudorandom numbers from a standard normal distribution. 


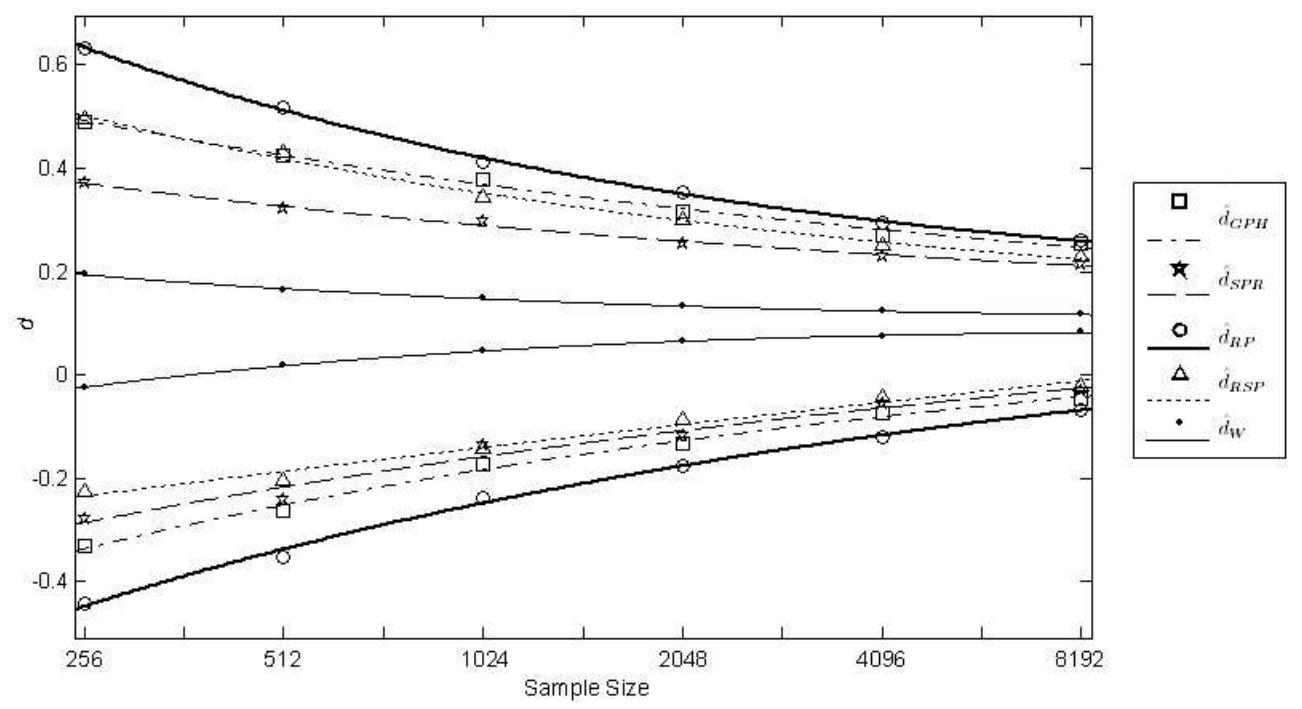

Fig. 3. Confidence interval for $d=0.10$ at $95 \%$ based on the five considered estimation methods and on six different sample sizes.

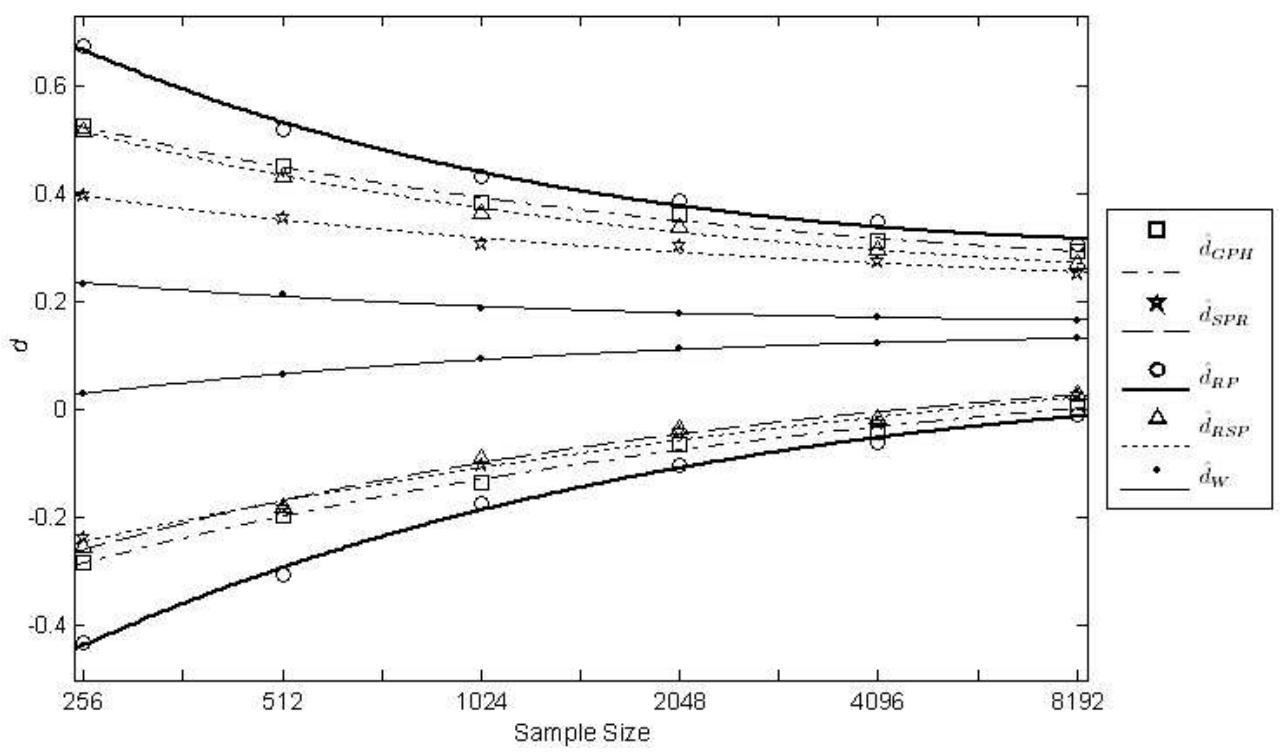

Fig. 4. Confidence interval for $d=0.15$ at $95 \%$ based on the five considered estimation methods and on six different sample sizes.

(3) Calculate the mean and the variance of the random variable $X_{t}$, where $X_{t}$ is obtained from (9) below.

(4) Generate a random variable $X_{t}$, with distribution $\mathcal{N}\left(m_{t}, v_{t}\right)$, for $t \in$ $\{1,2, \cdots, n\}$, where

$$
m_{t} \equiv \mathbb{E}\left(X_{t} \mid X_{\ell}, \ell<t\right)=\sum_{j=1}^{t} \varphi_{X}(t, j) X_{t-j}
$$




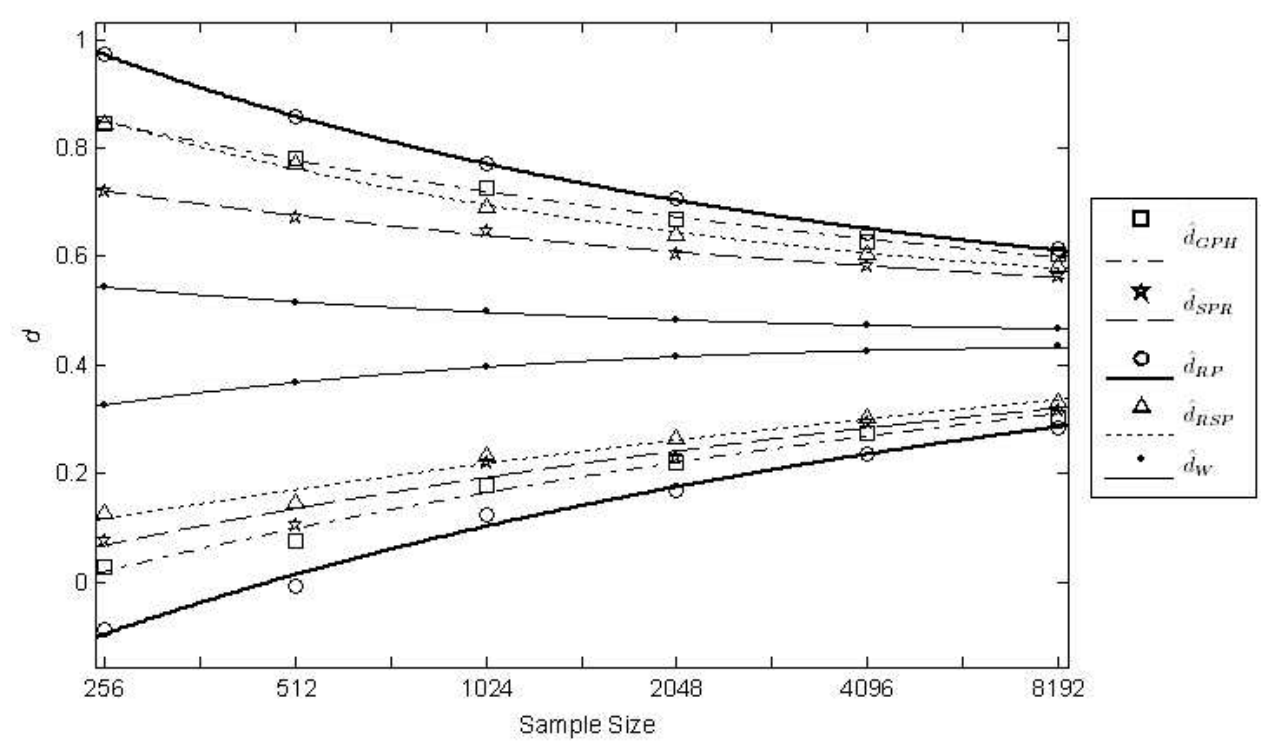

Fig. 5. Confidence interval for $d=0.45$ at $95 \%$ based on the five considered estimation methods and on six different sample sizes.

$$
v_{t} \equiv \operatorname{Var}\left(X_{t} \mid X_{\ell}, \ell<t\right)=\sigma_{\epsilon}^{2} \prod_{j=1}^{t}\left(1-\varphi_{X}^{2}(j, j)\right),
$$

$\varphi_{X}(t, j)$ is the partial autocorrelation of an $\operatorname{ARFIMA}(0, \mathrm{~d}, 0)$ process and $\sigma_{\epsilon}^{2}$ is the variance of the white noise process. For more details, see [22].

For the simulations of any $\operatorname{ARFIMA}(0, d, 0)$ process, we always use $\sigma_{\varepsilon}^{2}=1.0$ in expression (3). We observe that these processes are strongly stationary.

From the generation algorithm, given by (1) through (4), we obtain a sample time series $\left\{X_{t}\right\}_{t=1}^{n}$ from an $\operatorname{ARFIMA}(0, d, 0)$ process given by

$$
(1-\mathcal{B})^{d} X_{t}=\varepsilon_{t}, \text { for } t \in\{1,2, \cdots, n\}
$$

where the sample $\left\{X_{t}\right\}_{t=1}^{n}$ was obtained from the expression

$$
X_{t}=(1-\mathcal{B})^{-d} \varepsilon_{t}, \text { for } t \in\{1,2, \cdots, n\}
$$

The next step, after obtaining a time series, is to deal with the estimation of the fractional parameter $d$. In this work we use the estimators proposed in Section 4 , namely $\hat{d}_{G P H}, \hat{d}_{S P R}, \hat{d}_{R P}, \hat{d}_{R S P}$, and $\hat{d}_{W}$. For this we consider 1,000 time series. For each series we estimate the value of $d$ through the different methods and latter we take the arithmetic average of these values, that is, 
Table 1

The mean value and MSE, using different estimators, for different sample sizes $n$, based on $\operatorname{ARFIMA}(0, d, 0)$ with $d=0.05$.

\begin{tabular}{|c|c|c|c|c|c|}
\hline \hline \multicolumn{5}{|c|}{$d=0.05$} \\
\hline \multicolumn{5}{|c|}{ Estimation Method } \\
\cline { 2 - 6 } \multicolumn{5}{|c|}{ Mean Value } \\
\hline \hline$\hat{d}_{G P H}$ & $\hat{d}_{S P R}$ & $\hat{d}_{R P}$ & $\hat{d}_{R S P}$ & $\hat{d}_{W}$ \\
\hline \hline 256 & 0.0550 & 0.0091 & 0.0495 & 0.0460 & 0.0353 \\
512 & 0.0496 & 0.0176 & 0.0468 & 0.0456 & 0.0421 \\
1024 & 0.0530 & 0.0215 & 0.0544 & 0.0403 & 0.0446 \\
2048 & 0.0531 & 0.0340 & 0.0532 & 0.0493 & 0.0481 \\
4096 & 0.0515 & 0.0380 & 0.0529 & 0.0488 & 0.0481 \\
8192 & 0.0482 & 0.0369 & 0.0471 & 0.0441 & 0.0493 \\
\hline \hline \multicolumn{5}{|c|}{ MSE } \\
\hline \hline 256 & 0.0436 & 0.0273 & 0.0689 & 0.0384 & 0.0032 \\
512 & 0.0285 & 0.0182 & 0.0459 & 0.0233 & 0.0014 \\
1024 & 0.0167 & 0.0114 & 0.0237 & 0.0143 & 0.0007 \\
2048 & 0.0112 & 0.0074 & 0.0161 & 0.0089 & 0.0003 \\
4096 & 0.0078 & 0.0051 & 0.0098 & 0.0058 & 0.0002 \\
8192 & 0.0053 & 0.0034 & 0.0066 & 0.0037 & 0.0001 \\
\hline \hline
\end{tabular}

$$
\bar{d}_{i}=\frac{1}{1,000} \sum_{j=1}^{1,000} \widehat{d}_{i}(j),
$$

where $\bar{d}_{i}$ corresponds to $\hat{d}_{G P H}, \hat{d}_{S P R}, \hat{d}_{R P}, \hat{d}_{R S P}$, and $\hat{d}_{W}$, respectively, depending on the estimation method used. To compare the different estimators we considered the mean squared error value, denoted hereafter by MSE, i.e.,

$$
\mathrm{MSE}=\frac{1}{1,000} \sum_{j=1}^{1,000}\left(\widehat{d}_{i}(j)-d\right)^{2}
$$

where $d$ is the true parameter value.

In Tables 1-4 we present the simulation results for the fractional parameter $d \in\{0.05 ; 0.10 ; 0.15 ; 0.45\}$ in $\operatorname{ARFIMA}(0, d, 0)$ processes, for all estimation methods proposed here. 
Table 2

The mean value and MSE, using different estimators, for different sample sizes $n$, based on $\operatorname{ARFIMA}(0, d, 0)$ with $d=0.10$.

\begin{tabular}{|c|c|c|c|c|c|}
\hline \hline \multicolumn{5}{|c|}{$d=0.10$} \\
\hline \multirow{5}{*|c|}{ Estimation Method } \\
\cline { 2 - 6 } & $\hat{d}_{G P H}$ & $\hat{d}_{S P R}$ & $\hat{d}_{R P}$ & $\hat{d}_{R S P}$ & $\hat{d}_{W}$ \\
\hline \hline \multicolumn{5}{|c|}{ Mean Value } \\
512 & 0.1013 & 0.0684 & 0.1012 & 0.0967 & 0.0911 \\
1024 & 0.1091 & 0.0743 & 0.0941 & 0.0964 & 0.0951 \\
2048 & 0.1069 & 0.0790 & 0.0966 & 0.0972 & 0.0969 \\
4096 & 0.1035 & 0.0812 & 0.0970 & 0.0983 & 0.0986 \\
8192 & 0.1071 & 0.0844 & 0.1027 & 0.0951 & 0.0993 \\
\hline \hline & & 0.0656 & 0.1023 & 0.0931 & 0.0843 \\
\hline \hline 256 & 0.0434 & 0.0293 & 0.0711 & 0.0381 & 0.0033 \\
512 & 0.0299 & 0.0209 & 0.0426 & 0.0238 & 0.0015 \\
1024 & 0.0195 & 0.0127 & 0.0244 & 0.0135 & 0.0007 \\
2048 & 0.0111 & 0.0082 & 0.0169 & 0.0091 & 0.0003 \\
4096 & 0.0081 & 0.0052 & 0.0115 & 0.0067 & 0.0002 \\
8192 & 0.0047 & 0.0037 & 0.0061 & 0.0039 & 0.0001 \\
\hline \hline
\end{tabular}

We now construct empirical confidence intervals for the fractional parameter based on the estimation procedures given in Section 4. The process to construct the empirical confidence intervals consists of the following steps:

(1) For each sample size $n$ (we use $n \in\{256,512, \cdots, 8192\}$ ) we generate 1,000 replications;

(2) We calculate the lower bound $(0.5 \%, 2.5 \%$ e $5.0 \%)$ and upper bound $(99.5 \%, 97.5 \%$ e $95 \%)$ limits of the obtained estimator values. They are denoted by lower bound and upper bound, respectively;

(3) We construct a graph where the sample sizes are in the abscissa and the obtained values from the step 2 are in the ordinate axis. The values are fitted by a linear regression method using a MATLAB routine. From the fitted functions we construct the confidence intervals.

For instance, to get a confidence interval for parameter $d$ at $95 \%$ confidence level, we plot the values $2.5 \%$ and $97.5 \%$ of confidence interval versus the time series sample size $n$. For these data, the best adjusted function is 
Table 3

The mean value and MSE, using different estimators, for different sample sizes $n$, based on $\operatorname{ARFIMA}(0, d, 0)$ with $d=0.15$.

\begin{tabular}{|c|c|c|c|c|c|}
\hline \multicolumn{6}{|c|}{$d=0.15$} \\
\hline \multirow[t]{2}{*}{$n$} & \multicolumn{5}{|c|}{ Estimation Method } \\
\hline & $\hat{d}_{G P H}$ & $\hat{d}_{S P R}$ & $\hat{d}_{R P}$ & $\hat{d}_{R S P}$ & $\hat{d}_{W}$ \\
\hline \multicolumn{6}{|c|}{ Mean Value } \\
\hline 256 & 0.1502 & 0.0983 & 0.1502 & 0.1408 & 0.1327 \\
\hline 512 & 0.1404 & 0.1041 & 0.1428 & 0.1389 & 0.1418 \\
\hline 1024 & 0.1486 & 0.1167 & 0.1509 & 0.1407 & 0.1441 \\
\hline 2048 & 0.1495 & 0.1315 & 0.1493 & 0.1497 & 0.1475 \\
\hline 4096 & 0.1528 & 0.1347 & 0.1526 & 0.1480 & 0.1487 \\
\hline 8192 & 0.1527 & 0.1383 & 0.1534 & 0.1484 & 0.1494 \\
\hline \multicolumn{6}{|c|}{ MSE } \\
\hline 256 & 0.0436 & 0.0289 & 0.0751 & 0.0385 & 0.0031 \\
\hline 512 & 0.0295 & 0.0201 & 0.0475 & 0.0236 & 0.0015 \\
\hline 1024 & 0.0173 & 0.0120 & 0.0245 & 0.0133 & 0.0006 \\
\hline 2048 & 0.0121 & 0.0081 & 0.0164 & 0.0092 & 0.0003 \\
\hline 4096 & 0.0078 & 0.0054 & 0.0103 & 0.0059 & 0.0002 \\
\hline 8192 & 0.0054 & 0.0036 & 0.0065 & 0.0038 & 0.0001 \\
\hline
\end{tabular}

$$
h(n)=a\left[\log _{2}\left(\log _{2}(n)\right)^{2}\right]+b\left[\log _{2}\left(\log _{2}(n)\right)\right]+c,
$$

with coefficients $a, b$, and $c$ estimated by linear regression method using a MATLAB routine. Figures 2-5 present the confidence interval for different values of $d \in\{0.05 ; 0.10 ; 0.15 ; 0.45\}$ only at $95 \%$ confidence level, based on all estimation procedures considered here, with $n \in\{256,512, \cdots, 8192\}$. For other confidence levels, and other different values of $d$, the results are available upon request.

One observes in Figures 2-5 that the obtained values for the estimation of $d$ converge to the true parameter value as the sample size increases. This result was expected, since as long as the sample size increases, more precise will be the estimates for $d$, independently of the estimation procedure.

With the data used to construct the graph we can approximate functions that return the estimated value of $d$ for different sample sizes that we choose for the 
Table 4

The mean value and MSE, using different estimators, for different sample sizes $n$, based on $\operatorname{ARFIMA}(0, d, 0)$ with $d=0.45$.

\begin{tabular}{|c|c|c|c|c|c|}
\hline \hline \multicolumn{5}{|c|}{$d=0.45$} \\
\hline \multirow{5}{*|c|}{ Estimation Method } \\
\cline { 2 - 6 } & $\hat{d}_{G P H}$ & $\hat{d}_{S P R}$ & $\hat{d}_{R P}$ & $\hat{d}_{R S P}$ & $\hat{d}_{W}$ \\
\hline \hline \multicolumn{5}{|c|}{ Mean Value } \\
512 & 0.4601 & 0.4149 & 0.4545 & 0.4638 & 0.4436 \\
1024 & 0.4696 & 0.4344 & 0.4690 & 0.4730 & 0.4491 \\
2048 & 0.4569 & 0.4349 & 0.4552 & 0.4628 & 0.4497 \\
4096 & 0.4635 & 0.4452 & 0.4609 & 0.4664 & 0.4499 \\
8192 & 0.4583 & 0.4452 & 0.4583 & 0.4617 & 0.4501 \\
\hline \hline & & \multicolumn{5}{|c|}{ MSE } & 0.4391 \\
\hline \hline 256 & 0.0434 & 0.0284 & 0.0737 & 0.0366 & 0.0033 \\
512 & 0.0302 & 0.0211 & 0.0473 & 0.0242 & 0.0014 \\
1024 & 0.0190 & 0.0127 & 0.0264 & 0.0147 & 0.0007 \\
2048 & 0.0131 & 0.0090 & 0.0174 & 0.0096 & 0.0003 \\
4096 & 0.0086 & 0.0057 & 0.0106 & 0.0063 & 0.0002 \\
8192 & 0.0057 & 0.0039 & 0.0070 & 0.0041 & 0.0001 \\
\hline \hline
\end{tabular}

simulation. Table 5 supplies the confidence intervals for $d$ based on time series with sample size $n$ belonging to $\{256,512, \cdots, 8192\}$. With this procedure, we obtain the confidence interval for $d$ based on each estimation method. The upper and lower bounds of $d$ are calculated when we change $N$ to $\log _{2}\left(\log _{2}(n)\right)$ in the fitted equations of Tables 5-8.

Tables $1-4$ show the simulation results for $d \in\{0.05 ; 0.10 ; 0.15 ; 0.45\}$. One observes that, for the case where $d=0.10$, the best result is attained by $\hat{d}_{W}$.

\section{Application}

To test the effectiveness of the described procedure in Section 5, we analyze a real DNA sequence, calculating the confidence interval for all estimators pro- 
Table 5

Confidence intervals for $d=0.05$ at $95 \%$, where $N=\log _{2}\left(\log _{2}(n)\right)$, with different estimators.

\begin{tabular}{|c|c|c|}
\hline Estimator & Interval & Fitted Equation \\
\hline$\hat{d}_{G P H}$ & $\begin{array}{l}\text { upper bound } \\
\text { lower bound }\end{array}$ & $\begin{array}{c}h=0.1429 N^{2}-1.311 N+3.078 \\
h=-0.2635 N^{2}+2.160 N-4.485\end{array}$ \\
\hline$\hat{d}_{S P R}$ & $\begin{array}{l}\text { upper bound } \\
\text { lower bound }\end{array}$ & $\begin{array}{c}h=0.0335 N^{2}-0.438 N+1.308 \\
h=-0.1589 N^{2}+1.433 N-3.210\end{array}$ \\
\hline$\hat{d}_{R P}$ & $\begin{array}{l}\text { upper bound } \\
\text { lower bound }\end{array}$ & $\begin{array}{c}h=0.1704 N^{2}-1.654 N+3.978 \\
h=-0.4893 N^{2}+3.864 N-7.715\end{array}$ \\
\hline$\hat{d}_{R S P}$ & $\begin{array}{l}\text { upper bound } \\
\text { lower bound }\end{array}$ & $\begin{array}{c}h=0.1372 N^{2}-1.273 N+2.993 \\
h=-0.2678 N^{2}+2.198 N-4.547\end{array}$ \\
\hline$\hat{d}_{W}$ & $\begin{array}{l}\text { upper bound } \\
\text { lower bound }\end{array}$ & $\begin{array}{c}h=0.0931 N^{2}-0.724 N+1.470 \\
h=-0.1334 N^{2}+1.042 N-1.997\end{array}$ \\
\hline
\end{tabular}

Table 6

Confidence intervals for $d=0.10$ at $95 \%$, where $N=\log _{2}\left(\log _{2}(n)\right)$, with different estimators.

\begin{tabular}{|c|c|c|}
\hline \hline Estimator & Interval & Fitted Equation \\
\hline \hline$\hat{d}_{G P H}$ & upper bound & $h=0.0881 N^{2}-0.941 N+2.521$ \\
& lower bound & $h=-0.1491 N^{2}+1.419 N-3.250$ \\
\hline$\hat{d}_{S P R}$ & upper bound & $h=0.0711 N^{2}-0.704 N+1.843$ \\
& lower bound & $h=-0.0656 N^{2}+0.811 N-2.129$ \\
\hline$\hat{d}_{R P}$ & upper bound & $h=0.3447 N^{2}-2.845 N+6.068$ \\
& lower bound & $h=-0.2062 N^{2}+1.923 N-4.362$ \\
\hline$\hat{d}_{R S P}$ & upper bound & $h=0.1819 N^{2}-1.614 N+3.707$ \\
& lower bound & $h=0.0704 N^{2}-0.154 N-0.4058$ \\
\hline$\hat{d}_{W}$ & upper bound & $h=0.0921 N^{2}-0.731 N+1.553$ \\
& lower bound & $h=-0.1334 N^{2}+1.027 N-1.063$ \\
\hline \hline
\end{tabular}

posed in Section 4. We use available sequences in the National Center for Biotechnology Information (http://www.ncbi.nlm.nih.gov). Chosen a sequence, we use routines developed in this work to analyze it.

The first routine constructs a random walk (described in Section 3) for this sequence. From this random walk we use another routine developed in FORTRAN computational language (see [23] and [24]) to estimate $d$ based on the 
Table 7

Confidence intervals for $d=0.15$ at $95 \%$, where $N=\log _{2}\left(\log _{2}(n)\right)$, with different estimators.

\begin{tabular}{|c|c|c|}
\hline \hline Estimator & Interval & Fitted Equation \\
\hline \hline$\hat{d}_{G P H}$ & upper bound & $h=0.2032 N^{2}-1.691 N+3.768$ \\
& lower bound & $h=-0.1934 N^{2}+1.706 N-3.665$ \\
\hline$\hat{d}_{S P R}$ & upper bound & $h=0.1088 N^{2}-0.929 N+2.204$ \\
& lower bound & $h=-0.1170 N^{2}+1.168 N-2.699$ \\
\hline$\hat{d}_{R P}$ & upper bound & $h=0.5444 N^{2}-4.148 N+8.210$ \\
& lower bound & $h=-0.4586 N^{2}+3.679 N-7.348$ \\
\hline$\hat{d}_{R S P}$ & upper bound & $h=0.2434 N^{2}-1.977 N+4.256$ \\
& lower bound & $h=-0.2390 N^{2}+2.012 N-4.147$ \\
\hline$\hat{d}_{W}$ & upper bound & $h=0.0985 N^{2}-0.758 N+1.623$ \\
& lower bound & $h=-0.1305 N^{2}+1.021 N-1.860$ \\
\hline \hline
\end{tabular}

Table 8

Confidence intervals for $d=0.45$ at $95 \%$, where $N=\log _{2}\left(\log _{2}(n)\right)$, with different estimators.

\begin{tabular}{|c|c|c|}
\hline \hline Estimator & Interval & Fitted Equation \\
\hline \hline$\hat{d}_{G P H}$ & upper bound & $h=0.1145 N^{2}-1.123 N+3.187$ \\
& lower bound & $h=-0.0933 N^{2}+1.040 N-2.262$ \\
\hline$\hat{d}_{S P R}$ & upper bound & $h=0.0711 N^{2}-0.704 N+2.193$ \\
& lower bound & $h=-0.0716 N^{2}+0.842 N-1.813$ \\
\hline$\hat{d}_{R P}$ & upper bound & $h=0.2917 N^{2}-2.470 N+5.756$ \\
& lower bound & $h=-0.1900 N^{2}+1.818 N-3.840$ \\
\hline$\hat{d}_{R S P}$ & upper bound & $h=0.2435 N^{2}-2.018 N+4.713$ \\
& lower bound & $h=-0.0109 N^{2}+0.388 N-0.949$ \\
\hline$\hat{d}_{W}$ & upper bound & $h=0.0909 N^{2}-0.719 N+1.882$ \\
& lower bound & $h=-0.1779 N^{2}+1.343 N-2.102$ \\
\hline
\end{tabular}

estimation procedures proposed in Section 4.

In this section, the methodology is applied to the homo sapiens dystrophin sequence (muscular dystrophy, Duchenne and Becker types) (DMD, transcript variant Dp140bc, mRNA from NCBI \#NM 004023). For other applications of DNA sequences we refer the reader to [25]. 
The sequence of this example presents 7,048 nucleotides. Figure 6 shows the plot of the random walk for this DNA sequence. Table 9 shows the analysis result for all estimators proposed in Section 4 for this DNA sequence.

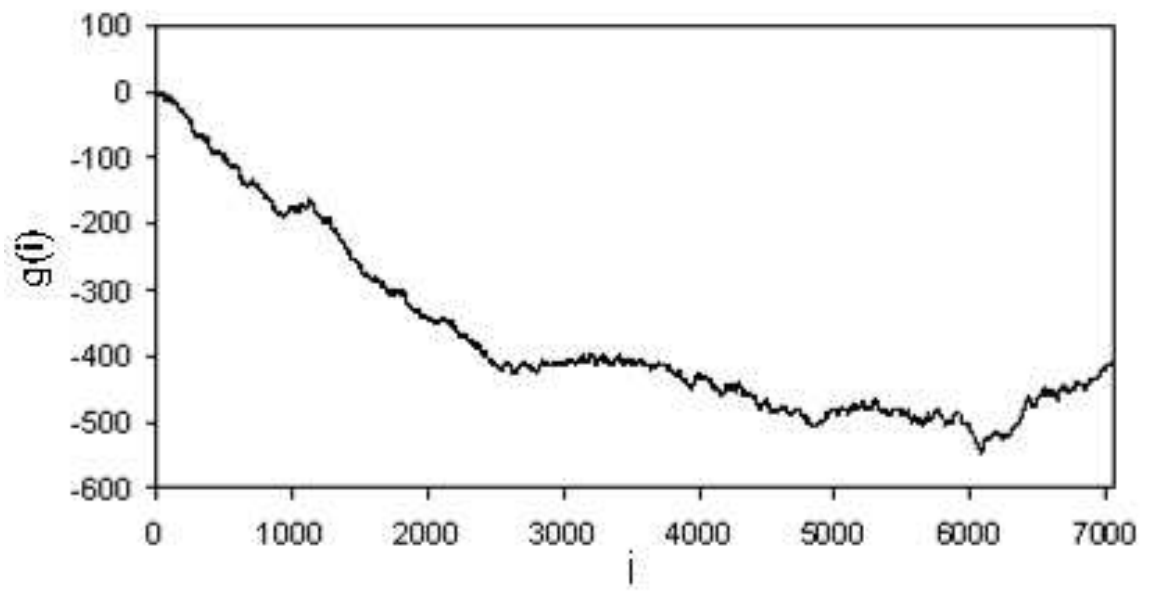

Fig. 6. Random walk for the homo sapiens dystrophin sequence.

Table 9

Estimation results for $d$ using different estimation methods.

\begin{tabular}{|c|c|c|c|c|c|}
\hline \hline Estimation Method & $\hat{d}_{G P H}$ & $\hat{d}_{S P R}$ & $\hat{d}_{R P}$ & $\hat{d}_{R S P}$ & $\hat{d}_{W}$ \\
\hline$\hat{d}_{i}$ value & 0.0922 & 0.0915 & 0.0902 & 0.0928 & 0.0930 \\
\hline \hline
\end{tabular}

Remark 7 The right choice of the number of regressors $g(n)=n^{\alpha}$ in expression (8) gave raise too many works among researchers and practitioners for the semiparametric estimation of $d$. For theoretical purpose, $g(n)$ is a function of $n$ such that $g(n) / n \rightarrow 0$, as $n \rightarrow \infty$. We refer the reader to [4], [9] and [17] for more details.

To calculate the confidence intervals for the estimators we use Table 6 . In this table one finds the fitted equations that allow to evaluate the upper and lower bounds of the estimators for the considered cases. The results are shown in Table 10 below.

Table 10

Results for the upper and lower bounds for the estimated value of $d$ using different estimation methods.

\begin{tabular}{|c|c|c|c|c|c|}
\hline \hline Estimation Method & $\hat{d}_{G P H}$ & $\hat{d}_{S P R}$ & $\hat{d}_{R P}$ & $\hat{d}_{R S P}$ & $\hat{d}_{W}$ \\
\hline upper bound & 0.2523 & 0.2158 & 0.2677 & 0.2319 & 0.1104 \\
\hline lower bound & -0.0485 & -0.0342 & -0.0794 & -0.0205 & 0.9097 \\
\hline \hline
\end{tabular}

According to Table 10 one observes that the estimated values for $d$ are in between the limits of the calculated fitted equations, at $95 \%$ confidence level (see the upper and lower bounds in Table 10). 
Table 11 presents the results for the estimation of the parameter $d$ for the homo sapiens dystrophin sequence, with random walk given in Figure 6 . The different estimation methods in the semiparametric class $\left(\hat{d}_{G P H}, \hat{d}_{S P R}, \hat{d}_{R P}\right.$, and $\left.\hat{d}_{R S P}\right)$ were calculated for different values of $\alpha$, where $g(n)=n^{\alpha}$ is the number of regressors given by expression (8) (see Remark 7). In this table we consider $\alpha \in\{0.50 ; 0.51 ; \cdots ; 0.95\}$ and it shows how the estimator value considerably changes with small changes in the $\alpha$-value. Table 11 also pre-

Table 11

Results for the estimation of $d$ using different values of $\alpha$.

\begin{tabular}{|c|c|c|c|c|c|c|c|c|c|}
\hline \multirow[t]{2}{*}{$\alpha$} & \multicolumn{5}{|c|}{ Estimation Method } & \multicolumn{4}{|c|}{ Absolute Error Value } \\
\hline & $\hat{d}_{G P H}$ & $\hat{d}_{S P R}$ & $\hat{d}_{R P}$ & $\hat{d}_{R S P}$ & $\hat{d}_{W}$ & $\hat{d}_{G P H}$ & $\hat{d}_{S P R}$ & $\hat{d}_{R P}$ & $\hat{d}_{R S P}$ \\
\hline 0.50 & 0.1513 & 0.1286 & 0.1137 & 0.0995 & 0.0930 & 0.0584 & 0.0356 & 0.0207 & 0.0065 \\
\hline 0.51 & 0.1485 & 0.1151 & 0.1136 & 0.0865 & 0.0930 & 0.0555 & 0.0221 & 0.0207 & 0.0065 \\
\hline 0.52 & 0.1231 & 0.0887 & 0.0878 & 0.0590 & 0.0930 & 0.0302 & 0.0042 & 0.0052 & 0.0340 \\
\hline 0.53 & 0.1296 & 0.0910 & 0.0979 & 0.0639 & 0.0930 & 0.0366 & 0.0020 & 0.0049 & 0.0291 \\
\hline 0.54 & 0.1355 & 0.0915 & 0.1071 & 0.0666 & 0.0930 & 0.0425 & 0.0015 & 0.0141 & 0.0264 \\
\hline 0.55 & 0.1672 & 0.1212 & 0.1448 & 0.1019 & 0.0930 & 0.0743 & 0.0283 & 0.0519 & 0.0089 \\
\hline 0.56 & 0.1632 & 0.1187 & 0.1421 & 0.1006 & 0.0930 & 0.0702 & 0.0258 & 0.0491 & 0.0076 \\
\hline 0.57 & 0.1551 & 0.1132 & 0.1350 & 0.0960 & 0.0930 & 0.0622 & 0.0202 & 0.0420 & 0.0030 \\
\hline 0.58 & 0.1686 & 0.1217 & 0.1513 & 0.1067 & 0.0930 & 0.0756 & 0.0288 & 0.0583 & 0.0137 \\
\hline 0.59 & 0.1477 & 0.1134 & 0.1299 & 0.0988 & 0.0930 & 0.0548 & 0.0205 & 0.0369 & 0.0059 \\
\hline 0.60 & 0.1475 & 0.1068 & 0.1311 & 0.0928 & 0.0930 & 0.0546 & 0.0138 & 0.0381 & 0.0002 \\
\hline 0.61 & 0.1673 & 0.1102 & 0.1538 & 0.0975 & 0.0930 & 0.0744 & 0.0173 & 0.0608 & 0.0046 \\
\hline 0.62 & 0.1498 & 0.0968 & 0.1359 & 0.0841 & 0.0930 & 0.0568 & 0.0039 & 0.0430 & 0.0089 \\
\hline 0.63 & 0.1358 & 0.0854 & 0.1219 & 0.0728 & 0.0930 & 0.0428 & 0.0076 & 0.0289 & 0.0202 \\
\hline 0.64 & 0.1282 & 0.0881 & 0.1149 & 0.0766 & 0.0930 & 0.0352 & 0.0049 & 0.0219 & 0.0164 \\
\hline 0.65 & 0.1157 & 0.0759 & 0.1026 & 0.0646 & 0.0930 & 0.0227 & 0.0170 & 0.0096 & 0.0284 \\
\hline 0.66 & 0.1093 & 0.0784 & 0.0967 & 0.0680 & 0.0930 & 0.0163 & 0.0146 & 0.0038 & 0.0250 \\
\hline 0.67 & 0.0897 & 0.0672 & 0.0769 & 0.0570 & 0.0930 & 0.0033 & 0.0258 & 0.0160 & 0.0360 \\
\hline 0.68 & 0.0946 & 0.0719 & 0.0831 & 0.0627 & 0.0930 & 0.0017 & 0.0211 & 0.0099 & 0.0303 \\
\hline 0.69 & 0.0825 & 0.0618 & 0.0712 & 0.0528 & 0.0930 & 0.0105 & 0.0312 & 0.0217 & 0.0402 \\
\hline 0.70 & 0.0821 & 0.0631 & 0.0716 & 0.0548 & 0.0930 & 0.0109 & 0.0299 & 0.0213 & 0.0382 \\
\hline 0.71 & 0.0747 & 0.0562 & 0.0647 & 0.0482 & 0.0930 & 0.0182 & 0.0368 & 0.0282 & 0.0448 \\
\hline 0.72 & 0.0762 & 0.0563 & 0.0670 & 0.0489 & 0.0930 & 0.0168 & 0.0367 & 0.0260 & 0.0441 \\
\hline 0.73 & 0.0761 & 0.0565 & 0.0676 & 0.0497 & 0.0930 & 0.0169 & 0.0365 & 0.0254 & 0.0433 \\
\hline 0.74 & 0.0473 & 0.0328 & 0.0383 & 0.0255 & 0.0930 & 0.0456 & 0.0602 & 0.0546 & 0.0675 \\
\hline 0.75 & 0.0444 & 0.0289 & 0.0359 & 0.0220 & 0.0930 & 0.0486 & 0.0641 & 0.0571 & 0.0709 \\
\hline 0.76 & 0.0413 & 0.0309 & 0.0334 & 0.0246 & 0.0930 & 0.0516 & 0.0620 & 0.0596 & 0.0683 \\
\hline 0.77 & 0.0483 & 0.0393 & 0.0411 & 0.0337 & 0.0930 & 0.0447 & 0.0537 & 0.0518 & 0.0592 \\
\hline 0.78 & 0.0442 & 0.0364 & 0.0374 & 0.0312 & 0.0930 & 0.0488 & 0.0565 & 0.0555 & 0.0617 \\
\hline 0.79 & 0.0446 & 0.0389 & 0.0384 & 0.0342 & 0.0930 & 0.0483 & 0.0540 & 0.0546 & 0.0588 \\
\hline 0.80 & 0.0533 & 0.0513 & 0.0478 & 0.0473 & 0.0930 & 0.0396 & 0.0416 & 0.0452 & 0.0457 \\
\hline 0.81 & 0.0515 & 0.0505 & 0.0463 & 0.0467 & 0.0930 & 0.0414 & 0.0425 & 0.0466 & 0.0463 \\
\hline 0.82 & 0.0556 & 0.0555 & 0.0509 & 0.0521 & 0.0930 & 0.0373 & 0.0375 & 0.0421 & 0.0409 \\
\hline 0.83 & 0.0673 & 0.0638 & 0.0632 & 0.0608 & 0.0930 & 0.0256 & 0.0292 & 0.0298 & 0.0322 \\
\hline 0.84 & 0.0638 & 0.0640 & 0.0599 & 0.0612 & 0.0930 & 0.0291 & 0.0289 & 0.0330 & 0.0317 \\
\hline 0.85 & 0.0661 & 0.0652 & 0.0625 & 0.0627 & 0.0930 & 0.0269 & 0.0277 & 0.0305 & 0.0303 \\
\hline 0.86 & 0.0681 & 0.0651 & 0.0648 & 0.0627 & 0.0930 & 0.0248 & 0.0279 & 0.0282 & 0.0303 \\
\hline 0.87 & 0.0745 & 0.0691 & 0.0716 & 0.0669 & 0.0930 & 0.0184 & 0.0238 & 0.0214 & 0.0260 \\
\hline 0.88 & 0.0809 & 0.0759 & 0.0782 & 0.0740 & 0.0930 & 0.0121 & 0.0170 & 0.0148 & 0.0189 \\
\hline 0.89 & 0.0816 & 0.0758 & 0.0791 & 0.0740 & 0.0930 & 0.0113 & 0.0172 & 0.0139 & 0.0190 \\
\hline 0.90 & 0.0834 & 0.0764 & 0.0811 & 0.0747 & 0.0930 & 0.0095 & 0.0166 & 0.0119 & 0.0183 \\
\hline 0.91 & 0.0884 & 0.0835 & 0.0863 & 0.0820 & 0.0930 & 0.0045 & 0.0095 & 0.0067 & 0.0110 \\
\hline 0.92 & 0.0922 & 0.0882 & 0.0902 & 0.0869 & 0.0930 & 0.0008 & 0.0048 & 0.0027 & 0.0061 \\
\hline 0.93 & 0.0918 & 0.0880 & 0.0898 & 0.0866 & 0.0930 & 0.0012 & 0.0050 & 0.0031 & 0.0063 \\
\hline 0.94 & 0.0955 & 0.0841 & 0.0883 & 0.0856 & 0.0930 & 0.0025 & 0.0089 & 0.0047 & 0.0074 \\
\hline 0.95 & 0.0978 & 0.0879 & 0.0869 & 0.0876 & 0.0930 & 0.0048 & 0.0051 & 0.0061 & 0.0054 \\
\hline
\end{tabular}


sents the absolute error value for each estimator in the semiparametric class compared with the $\hat{d}_{W}$ estimator. The smallest absolute error value, for each semiparametric method, is in bold faced character. The estimation values in Table 11 with the corresponding smallest absolute error value are the ones given in Table 9.

\section{Conclusion}

In this work we analyzed five different estimators for the long memory parameter $d$. From all these analyzed estimators, we can observe that $\hat{d}_{W}$ is the estimator that better behaves. Tables 1, 3, and 4 and Figures 2, 4, and 5 show that $\hat{d}_{W}$ has lesser variation among the maximum, and minimum values. Also, $\hat{d}_{W}$ has the smallest mean squared error, as we can see in Tables 1, 3, and 4. The best estimation procedure, in the statistical sense, is the maximum likelihood method, hereafter denoted by $\hat{d}_{W}$. It is always asymptotically unbiased and normally distributed (see [4], [9], [18] and [26]). The semiparametric methods $\left(\hat{d}_{G P H}, \hat{d}_{S P R}, \hat{d}_{R P}\right.$, and $\left.\hat{d}_{R S P}\right)$ are easier to be implemented and even more flexible, but if one has the information that the data comes from an ARFIMA model, then the right method to be used is the maximum likelihood procedure. This is in accordance with the simulation results obtained in the tables.

A question can be asked from the results when we use real data. Which estimator is the best choice when analyzing DNA sequences? As we noted in the previous paragraph, $\hat{d}_{W}$ has lesser variation among the maximum, and minimum values and the smallest mean squared error value. So, if we have to choose a method to estimate the parameter $d$ in a time series, obtained from an $\operatorname{ARFIMA}(0, d, 0)$ process, we must choose the $\hat{d}_{W}$ method.

One can also note that $\hat{d} \in(0.0,0.5)$ for all methods. With this result, we conjecture that DNA sequences have long range dependence. However, the $\hat{d}_{W}$ estimator has slow convergence. When one is dealing with large size of observations in a time series (for instance, DNA sequences with more than 4,000 base pairs), the $\hat{d}_{W}$ estimator takes some minutes to converge, while the other estimators $\left(\hat{d}_{G P H}, \hat{d}_{S P R}, \hat{d}_{W}, \hat{d}_{R P}\right.$, and $\left.\hat{d}_{R S P}\right)$ converge more quickly. We believe that this fact is due to the FORTRAN routines used in this work, since they were not optimized to a statistical use.

\section{Acknowledgements}

S.R.C. Lopes was partially supported by CNPq-Brazil, by Pronex Probabili- 
dade e Processos Estocásticos (Convênio MCT/CNPq/FAPERJ - Edital 2003) and also by Fundação de Amparo à Pesquisa no Estado do Rio Grande do Sul (FAPERGS). The authors would like to thank to the editor and one anonymous referee for the valuable comments and suggestions that improved this version of the manuscript.

\section{References}

[1] W. Li and K. Kaneko, Long-Range Correlation and Partial $1 / f^{\alpha}$ Spectrum in a Noncoding DNA Sequence, Europhysics Letters 17 (7) (1992) 655-660.

[2] M. Osaka, K. Gohara, S. Ishii, H. Kishida, H. Hayakawa, and N. Ito, Symbolic Strings and Spatial 1/f spectra, Physica D 125 (1-2) (1999) 142-154.

[3] B. Borstnik, D. Pumpernik and D. Lukman, Analysis of Apparent $1 / f^{\alpha}$ Spectrum in DNA Sequences, Europhysics Letters 23 (6) (1993) 389-394.

[4] J. Beran, Statistics for Long Memory Processes (Chapman \& Hall, New York, 1994).

[5] H.E. Hurst, Long-Term Storage Capacity of Reservois, Trans. Amer. Soc. Civil Eng. 116 (1951) 770-799.

[6] C.W.J. Granger and R. Joyeux, An introduction to long-memory time series models and fractional differencing, Journal of Time Series Analysis 1 (1980) $15-30$.

[7] J.R.M. Hosking, Fractional Diferencing, Biometrika 68 (1) (1981) 165-176.

[8] J.R.M. Hosking, Modelling persistence in hidrological time series using fractional differencing. Water Resources Research 20 (12) (1984) 1898-1908.

[9] P.J. Brockwell and R.A. Davis, Time Series: Theory and Methods (SpringerVerlag, New York, 1991).

[10] H.E. Stanley, S.V. Buldyrev, A.L. Goldberger, S. Havlin, C.K. Peng, and M. Simons, Scaling features of noncoding DNA, Physica A 273 (1) (1999) 1-18.

[11] C. Peng, S.V. Buldyrev, A.L. Goldberger, S. Havlin, F. Sciortino, M. Simons, and H.E. Stanley, Long-range correlations in Nucleotide Sequences, Nature 356 (1992) 168-170.

[12] Z.G. Yu, V.V. Anh, and B. Wang, Correlation property of length sequences based on global structure of the complete genome, Physical Review E 63 (2000) (011903).

[13] B.P. Olbermann, S.R.C. Lopes, and V.A. Reisen, Invariance of the First Difference in ARFIMA Models, Accepted for publication in Computational Statistics, 2005. 
[14] S.R.C Lopes, B.P. Olbermann, and V.A. Reisen, A Comparison of Estimation Methods in Non-stationary Arfima Processes, Journal of Statistical Computation and Simulation 74 (5) (2004) 339-347.

[15] J. Geweke and S. Porter-Hudak, The estimation and application of long-memory time series models, Journal of Time Series Analysis 4 (4) (1983) 221-238.

[16] V.A. Reisen, Estimation of the Fractional Difference Parameter in the ARIMA $(p, d, q)$ model using the Smoothed Periodogram, Journal of Time Series Analysis 15 (3) (1994) 335-350.

[17] P.M. Robinson, Log-Periodogram Regression of Time Series with Long Range Dependence, The Annals of Statistics 23 (3) (1995) 1040-1072.

[18] R. Fox and M. Taqqu, Large sample properties of parameter estimates for strongly dependent stationary time series, The Annals of Statistics 14 (1986) 517-532.

[19] P. Whittle, Estimation and information in stationary time series, Arkiv für Mathematik 2 (1953) 423-434.

[20] M.S. Taqqu, V. Teverovsky, and W. Willinger, Estimators for long-range dependence: an empirical study, Fractals 3 (4) (1995) 785-798.

[21] R. Weron, Estimating long-range dependence: finite sample properties and confidence intervals, Physica A 312 (1-2) (2002) 285-299.

[22] C. Bisognin and S.R.C. Lopes, Estimating the Long Memory Parameter in the Presence of Seasonality. Submitted.

[23] V.A. Reisen and S.R.C. Lopes, Some Simulations and Applications of Forecasting Long Memory Time Series Models, Journal of Statistics and Planning Inference 80 (2) (1999) 269-287.

[24] V.A. Reisen, B. Abraham, and S.R.C. Lopes, Estimation of Parameters in ARFIMA Processes: A Simulation Study, Communications in Statistics: Simulation and Computation 30 (4) (2001) 787-803.

[25] M.A. Nunes and S.R.C. Lopes, Análise de Longa Dependência em Seqüências de DNA, Technical Report, Serie A, No. 60, Instituto de Matemática, UFRGS, Porto Alegre (2004).

[26] F. Sowell, Maximum Likelihood Estimation of Stationary Univariate Fractionally Integrated Time Series Models, Journal of Econometrics 53 (1992) 165-188. 